\title{
Coverillustration
}

\section{Through a lens sharply}

$\mathrm{T}$ he blue fin tuna (Thunnus thynnus) is a wide ranging pelagic predator and vastly overexploited food resource with enviable visual mechanisms. As can be seen on the cover, the blue fin tuna provides succulent sashimi, but the animal is extraordinary in several other aspects as well.

Most fish are poikilothermic, and as a result, maintain the temperature of the sea around them. The lower body temperature may penalise such fish because sluggishness and neurological lethargy will accompany lower body temperatures. A poikilothermic predator, then, risks temperature induced languor, as well as poorer optical function during the hunt. To solve this problem, some piscine predators have evolved ingenious thermal mechanisms. The blue fin tuna, nearly at the apex of the food chain, has solved this problem in a curious manner.

Blue fin tuna are not poikilothermic, but endothermic, at least partially. The predatory tuna species will sustain body core temperatures that average $23-26^{\circ} \mathrm{C}$; a core temperature which is sometimes as much as $21^{\circ} \mathrm{C}$ above ambient temperatures. These are not mammalian levels, to be sure, but they are sufficient to ensure that these predators will be spry in colder waters, especially when compared to their prey. These scombroid fish accomplish this remarkable feat with several specialised features including a countercurrent heat exchange, enabling them to isolate the core of their bodies thermally, and eliminate the loss of heat at their gills and external body surfaces (Altringham JD, Block BA, J Exp Biol 1997;200:2617-27).

Other fish, including billfishes such as the swordfish (BJO cover, March 2002), some sharks, and certain species of mackerel, have some features of endothermy although the strategies of each are different. This would suggest that this interesting ability has evolved at least three separate times in teleosts. Each time this evolution coincided with the species moving into colder waters (Block BA, Carey FR, J Comp Physiol (B) 1985;156:229-36). This would provide a locomotory advantage over any prey species that could not warm its musculature, brain, and visual mechanisms.

The blue fin tuna may range 25000 miles or more during a year, and will dive to depths of up to 1000 metres. It must have superb visual mechanisms to accomplish these feats. The increased body temperature of the blue fin (and other species of tuna) will improve muscular activity by increasing endurance and enhancing power output for both cruising and bursts of speed.

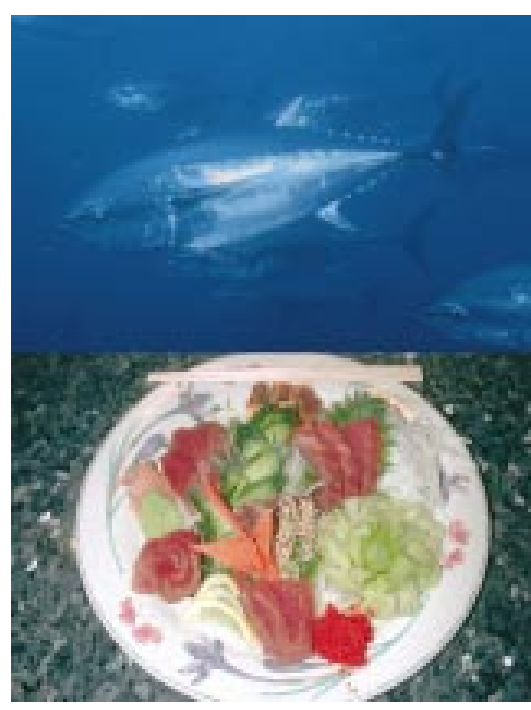

The blue fin tuna can be a very large fish, weighing up to $750 \mathrm{~kg}$, and as a consequence, sports an enormous crystalline lens that is spectacular-perhaps as large as 2.5 centimetres or more in diameter. A lens of this size risks spherical aberration as the periphery of the lens supports light transmission and most piscine pupils are fixed at approximately the maximum diameter of the lens. Hence, the peripheral portion of such a lens may be responsible for transmission of light rays to form an image. Spherical aberration would be significant if the lens were of one uniform index of refraction. This is especially important since any fish utilising aquatic vision will lose the refractive ability of its cornea as the refractive index of cornea and water are almost the same. Aquatic vision requires that the crystalline lens be almost entirely responsible for the total dioptric power of the eye. Spherical aberration would represent a significant visual problem requiring very special lenses.

The tuna's interesting solution is a lens with a decreasing index of refraction beginning with a higher index of refraction in the nucleus and declining towards the periphery. This declination of the index of refraction from centre to periphery is seen in other fish and even non-aquatic animals as well, but the tuna has one of the largest of crystalline lenses and must face spherical aberration with special dread because of this lens size. (This declination of the index of refraction was documented in Haplochromis burtoni, an African cichlid by Fernald RD (Nature 1983;301:618-20)).

The spherical crystalline lens will grow throughout life, and may increase 1000 -fold in size during life, in some species. As the lens grows and adds new lens fibres peripherally, the more central layers slowly lose their water content and the fibres become more compact. The index of refraction of the peripheral lens fibres is approximately 1.38 compared to the maximum of 1.56 as it becomes more compact (Fernald RD, Nature 1983;301:618-20). This clever dynamic mechanism will virtually eliminate spherical aberration, even in a large crystalline lens.

An additional interesting aspect of this tangerine-sized eye is its scleral ossicles. Many fish, especially the larger, swifter, and more deep diving ones, such as tuna, have additional support to the anterior portion of the globe. The tuna has two in the form of demilunar ossicles embedded in the connective tissue of the sclera. The tuna has among the heaviest periocular bones that join to form a complete "napkin ring" around the whole anterior segment of the globe, although these are probably not related evolutionarily to those of Ophthalmosaurus (BJO cover, Feb 2002).

The blue fin tuna, then, is an endothermic marvel of the piscine world, having one of the best crystalline lens optics with the sharpest focus in the entire vertebrate world. It is also a sumptuous feast of sashimi in the mammalian world.

Ivan R Schwab,

University of California Davis, Department of Ophthalmology, $4860^{\circ}$ "Y" Street, Suite 2400,
Sacramento, CA 95817, USA; irschwab@ucdavis.edu

Cover image of blue fin tuna with copyright taken by Richard Herrmann/Seapics.com, Hawaii, and the photograph of the plate of sashimi is by the author. The sashimi was prepared by chef Taro of the Mikuni Restaurant in Fair Oaks, CA, USA. (It was delicious!) 